\title{
Zolmitriptan for Early Onset of Action in Acute Migraine Attacks
}

\author{
Stephanie J Nahas \\ Department of Neurology, Thomas Jefferson University, Philadelphia, PA, USA
}

DOI: https://doi.org/10.17925/USN.2021.17.2.74

$\mathrm{M}$ igraine is a common, disabling and costly disease affecting $12 \%$ of the global population. It is typified by recurrent attacks of head pain and associated symptoms lasting hours to days. There are many aspects to managing migraine disease, but common to all patients with migraine is the need for treatment to stop an attack once it has begun. Many therapeutic options exist, with a wide range of mechanisms, tolerability and efficacy. Rapidity of relief is critical. Zolmitriptan, a 5-hydroxytryptamine 1B/1D receptor agonist, has been approved by the US Food and Drug Administration for the treatment of migraine attacks since 1997. It is available in multiple formulations, with a new one in development. This review covers the role zolmitriptan plays, and may play in the future, in treating migraine attacks.

\section{Keywords}

Migraine, triptan, zolmitriptan,

pharmacology, intranasal, intracutaneous, acute migraine treatment

Disclosure: Stephanie J Nahas has received honoraria for consulting from: Alder/Lundbeck, Allergan/AbbVie, Amgen/Novartis, Axsome, BioDelivery Sciences,

Biohaven, Fli Lilly, Fenix Group International, Impel, Nesos Corp (formerly Vorso Corp), Teva; honoraria for speaking from: Allergan/AbbVie, Amgen/Novartis, Eli Lilly, Teva (these relationships are now inactive); honoraria for work in education or publishing from: American Academy of Neurology, American Headache Society, Evolve Med Ed, Massachusetts Medical Society, MedLink Neurology, MJH Life Sciences, NACCME, Neurology Learning Network, Pennsylvania Neurologic Society, Springer, WebMD/Medscape, Wolters-Kluwer; and legal fees for serving as a medical expert to Jackson \& Campbell.

Review process: Double-blind peer review.

Compliance with ethics: This article involves a review of the literature and did not involve any studies with human or animal subjects performed by any of the authors.

Data availability: Data sharing is not applicable to this article as no datasets were generated or analysed during the writing of this article.

Authorship: The named author meets the Internationa Committee of Medical Journal Editors (ICMJE) criteria for authorship of this manuscript, takes responsibility for the integrity of the work as a whole, and has given final approval for the version to be published.

Access: This article is freely accessible at

touchNEUROLOGY.com. (C) Touch Medical Media 2021

Received: 21 October 2021

Accepted: 6 December 2021

Published online: 21 December 2021

Citation: touchREVIEWS in Neurology. 2021;17(2):74-6

Corresponding author: Stephanie J Nahas, Department of Neurology, Thomas Jefferson University, Jefferson Headache Center, 900 Walnut St., Suite 200, Philadelphia, PA 19107-5509, USA. E: Stephanie.Nahas@jefferson.edu

Support: No funding was received for

the publication of this article.
Migraine is a neurologic disease characterized by recurrent attacks of head pain and symptoms such as nausea, photophobia and phonophobia, which last hours to days. ${ }^{1}$ Attacks themselves can be disabling, and due to the often unpredictable and unavoidable nature of the attacks, the disease itself accounts for substantial disability. ${ }^{2}$ In fact, migraine affects $12 \%$ of the global population and is the number one cause of years lived with disability among Americans aged 15-49 years. ${ }^{2}$ In addition, migraine disease accounts for an estimated $\$ 30$ billion in direct and indirect costs annually in the US. ${ }^{3}$ Every individual with migraine needs a management strategy to stop an attack quickly after onset. In most cases, this involves taking medication, and a wide variety of options are available. For best results, treatment should be taken as soon as possible after the attack has started, and should have as rapid an onset of action as possible. ${ }^{4}$ The US Food and Drug Administration (FDA) has approved several medications for this purpose, including non-specific medications such as analgesics, as well as disease-specific medications such as ergots, triptans, gepants and ditans. These therapies vary greatly in terms of their mechanism of action, tolerability, safety, efficacy and speed of onset. ${ }^{4}$ One of these options, zolmitriptan, a 5-hydroxytryptamine 1B/1D receptor agonist, has been FDA-approved for clinical use in adults with migraine since 1997, ${ }^{5}$ and in 2015, the FDA expanded the 2003 indication for the intranasal formulation to include adolescents aged 12-17 years. ${ }^{6}$ Currently, it is available in oral tablets, orally disintegrating tablets and nasal spray, in doses of $2.5 \mathrm{mg}$ and $5 \mathrm{mg}$ in each case. ${ }^{5,6} \mathrm{In}$ development is another formulation using an adhesive, dermally applied, microneedle-array patch to deliver intracutaneous zolmitriptan..$^{-9}$ An overview of the management of migraine attacks and the properties of zolmitriptan for said use are discussed.

\section{What makes for an ideal acute migraine attack medication?}

several things are to be considered when envisioning an optimized medication for migraine attacks. ${ }^{4}$ As mentioned above, a rapid onset of action is critical to stopping the attack as soon as possible. Indeed, in several survey studies, this property is considered to be the most important by clinicians and patients alike. ${ }^{4}$ The rapidity of onset depends on several factors. A brief review of core pharmacologic principles follows. ${ }^{10}$

First, the drug must enter the systemic circulation. The route of administration has great influence on this. Orally administered medications are absorbed at the slowest rate. This is due to the inherent nature of gastric and intestinal absorption, but can be further influenced by phenomena such as the lipophilicity of the drug, P-glycoprotein activity, interaction with food and changes in gastric motility during a migraine attack. The greater the lipophilicity of a drug, the faster it is absorbed. P-glycoprotein activity, on the other hand, can pump a drug out of a cell, leading to reduced overall absorption. Certain foods may affect gastric absorption. When gastric stasis occurs during a migraine attack, this further reduces the efficiency of drug absorption. Furthermore, once a drug is absorbed through the gastrointestinal system, first-pass hepatic metabolism may influence the rate of rise in concentration of the drug. To bypass these obstacles, not only can drugs be designed to have greater oral absorption based on their chemical properties, they can be administered via alternative routes..$^{10}$ 
Rectally administering a drug not only bypasses potential obstacles of gastric acid, gastric stasis, interference from food and loss of drug from vomiting, it allows for enhanced systemic delivery since the rectum is more richly vascularized and primed for absorption compared with the upper gastrointestinal tract. Hepatic first-pass metabolism still occurs with rectally administered drugs, but it is reduced to about onethird of that of orally administered drugs, since about two-thirds of the venous drainage from the rectum is systemic rather than portal hepatic. Acceptance and use of rectally administered drugs is highly cultural, and relatively low in the US. ${ }^{10}$

A medication may be formulated for topical administration via the oral mucosa (sublingual/buccal). This confers all the advantages of rectal administration, with the additional benefit of the complete elimination of first-pass metabolism. Few medications are currently available in this form. ${ }^{10}$

Similarly to buccal administration, intranasal administration allows for rapid absorption while bypassing first-pass metabolism. However, some of the drug will inevitably be swallowed before it can be absorbed through the nasal mucosa, thus leading to some variability in pharmacokinetic curves. For centrally acting drugs, the concept of nose-to-brain delivery applies, wherein a drug is not only absorbed systemically through the mucosa into the bloodstream, but may also be delivered directly to the brain through olfactory and trigeminal pathways. This is achieved only through the upper-nasal space, an area which is difficult to access via traditional liquid nasal-spray delivery systems. The importance of this in managing acute migraine attacks has been debated, but has spurred the development of specialized devices intended to deposit drugs primarily into this cavity. ${ }^{11}$

The transdermal route similarly bypasses first-pass metabolism, but requires the drug to be lipophilic. Furthermore, there can be great variability in the rate and degree of absorption based on the location, thickness and health of the skin. Transdermal absorption can be hastened by using electrical current to induce iontophoresis. ${ }^{10}$ The only such acute migraine attack therapy which came to market, the sumatriptan iontophoretic patch, while effective, had to be withdrawn from the market due to skin burns in some patients. ${ }^{12}$

Intracutaneous delivery is similar to transdermal, with the advantage of direct penetration through the stratum corneum, thus allowing for faster and more reliable absorption through the capillary beds. An additional advantage is that this can be achieved through a drug-coated microneedle-array patch, which produces minimal to no pain or bleeding compared with a traditional needle. ${ }^{13}$ Currently, there are no available drugs of any sort delivered in this fashion. ${ }^{10}$

Subcutaneous delivery completely bypasses the stratum corneum, thus avoiding some of the limitations of transdermal delivery. Injection site pain and needle phobia are the main barriers to its use. Similarly, intramuscular delivery is comparable to the subcutaneous route in terms of absorption into the bloodstream. Injection site pain tends to be greater by comparison, and injection technique is more complicated, posing further barriers to its use..$^{10}$

Intravenous administration allows for near instantaneous absorption and $100 \%$ bioavailability, but typically requires specialized medical care to deliver. ${ }^{10}$

Delivery through the lungs via oral inhalation is nearly as efficient as directly through the vein, with the added advantages that it's painless and requires no special training, monitoring or setting to administer. ${ }^{10}$ Only one anti-migraine agent entered the development stage for such administration before it faltered in the final stages of optimization of the inhaler device, and has since been abandoned. ${ }^{14}$

Once a drug enters the systemic circulation, it needs to access its target of its mechanism of action, and remain in sufficient concentration for long enough to stop the attack. This process is influenced by proteinbinding capacity, volume of distribution, speed of metabolism and elimination from the body. For the purposes of rapid onset of action of a drug, these considerations are secondary to how quickly the drug can get into the body in the first place. In other words, ideally, a medication reaching its effective concentration rapidly is not only dependent on the rate of absorption but also on the rate of elimination. ${ }^{10}$

Another consideration is that some medications have subsequent effects due to active metabolites or activation of secondary pathways. This is relevant in migraine, as several drugs used to treat acute attacks do indeed have active metabolites, and their pharmacokinetics can depend on all of the above and more. Furthermore, given the complex and still mysterious nature of migraine, it is possible that there are downstream phenomena of the attack itself, as well as the medication used to treat it, that are important to consider. ${ }^{15}$

There may be a price to pay for attempting to optimize drug delivery for rapid relief. First, as noted above, some of the more rapidly delivered formulations are unseemly for some patients, and even painful. Second, a rapid rise to effective concentration tends to result in 'overshoot', i.e. a further rapid rise to supratherapeutic concentrations, which may be associated with more intense adverse events. Third, one strategy for ensuring a rapid concentration rise necessitates impeding degradation, resulting in longer exposure and greater areas under the curves, which also makes adverse events more likely. Finally, it remains somewhat debatable how, and to what extent, pharmacokinetic differences may influence medication effectiveness. ${ }^{10}$

Currently, zolmitriptan can be administered by the oral or intranasal routes only. The orally disintegrating tablet is not absorbed through the oral mucosa, but rather after swallowing the solution created when it dissolves in saliva. The pharmacokinetics of these oral formulations are very similar. Intranasal delivery is demonstrably faster. Intracutaneous delivery is in development. ${ }^{-9}$

\section{Oral zolmitriptan}

Oral zolmitriptan was patented in 1990 and first approved for the acute treatment of migraine attacks in adults via oral dosing $(2.5 \mathrm{mg}$ or $5 \mathrm{mg}$ ) by the FDA in $1997 .{ }^{5}$ It came to market with potential advantages compared with sumatriptan, which was the first triptan approved by the FDA in $1992 .{ }^{16}$ Zolmitriptan is more lipophilic than sumatriptan, and the oral bioavailability is about $40 \%$ (for sumatriptan, oral bioavailability is about 15\%). Zolmitriptan also has an active metabolite, N-desmethylzolmitriptan, whereas sumatriptan does not. Protein binding and elimination half-life are similar between the two drugs, and drug interactions with monoamine oxidase inhibitors are possible with both sumatriptan and zolmitriptan. ${ }^{17}$ A meta-analysis compared attributes of six of the seven FDA-approved triptans for migraine, at a time when zolmitriptan was available only in oral form. ${ }^{18}$ Despite some inherent differences between zolmitriptan and sumatriptan, as mentioned above, oral zolmitriptan $2.5 \mathrm{mg}$ and $5 \mathrm{mg}$ were considered comparable to oral sumatriptan $100 \mathrm{mg}$ in terms of efficacy, tolerability and consistency of effect across multiple attacks. ${ }^{18}$ 
After oral ingestion, zolmitriptan is detectable in serum after about 15-20 minutes, and the active metabolite may be detected very soon thereafter. ${ }^{17}$ The plasma concentration-time profiles of parent drug and metabolite are nearly identical with oral dosing. ${ }^{19}$ Both reach maximal plasma concentration within about 2 hours followed by a slow drop in concentration thereafter; the elimination half-life is just under 3 hours for each. ${ }^{17}$

\section{Intranasal zolmitriptan}

The intranasal formulation of zolmitriptan was approved by the FDA in 2003. The intranasal doses are the same as the oral doses, $2.5 \mathrm{mg}$ and $5 \mathrm{mg} .{ }^{20}$ As expected, serum levels are detectable much sooner than after an oral dose (within 2 minutes), and at 10 minutes the concentration is about one-third of the peak concentration. ${ }^{19}$ A nuclearimaging study demonstrated that nearly $100 \%$ of the dose is present in the nasopharynx immediately after administration, with a slow drop-off thereafter. ${ }^{21} \mathrm{~A}$ negligible amount is present in the lungs. Zolmitriptan is detectable in brain tissue within 5 minutes of an intranasal dose, further supporting rapid delivery to the target.22 However, due to bypassing portal circulation, for the most part, the appearance of the active metabolite is delayed by about 15 minutes compared with oral dosing. ${ }^{19}$ some argue that this phenomenon offers a 'two-tiered' approach to the migraine attack with the intranasal formulation. Considering the rapid rise in serum concentration, delayed appearance of the active metabolite and lack of pulmonary absorption, one may conclude that a substantial portion of the dose is absorbed directly across the nasal mucosa. To support this, a study comparing oral and intranasal zolmitriptan, with and without activated charcoal, was performed. Oral absorption is nearly completely blocked by activated charcoal, and for intranasal administration, serum concentrations are blunted by simultaneous oral ingestion of activated charcoal. It is estimated that about $30 \%$ of total exposure to the drug is due to nasal absorption after intranasal delivery. ${ }^{23}$ Despite differing shapes to the concentration-time curves, total exposure to zolmitriptan via oral and intranasal routes, estimated by areas under the curves, was nearly identical, suggesting equal bioavailability between the two formulations, which is interesting and difficult to explain fully. ${ }^{23}$

\section{Intracutaneous zolmitriptan}

As mentioned previously, intracutaneous delivery of zolmitriptan is in development. An early pharmacokinetic study in pigs demonstrated a high absolute bioavailability of $77 \%$ and a short time to maximal concentration of about 15 minutes. ${ }^{7}$ The drug-coated microprojections penetrated the skin at an average depth of $150 \mu \mathrm{m}$. Concentration-time curves for intravenous and intracutaneous administration overlapped at about 45 minutes, and remained overlapping thereafter. Transient mild-to-moderate skin erythema was observed after intracutaneous delivery, as can be expected with this modality.

In human studies of intracutaneous zolmitriptan at a dose of $3.8 \mathrm{mg}$ (two $1.9 \mathrm{mg}$ patches) for migraine, efficacy, tolerability and safety were favorable in both a randomized, double-blind, placebo-controlled clinical trial and a long-term safety study. ${ }^{8.9}$ Efficacy appears comparable to that of oral and intranasal zolmitriptan..$^{18}$ In the pivotal study, treatment-related adverse events were reported in $16.9 \%$ of participants receiving placebo and in up to $50.6 \%$ of participants receiving active treatment. ${ }^{8}$ The majority of these reactions were mild and dermal in nature. Systemic adverse events were less commonly reported, even more rarely than in initial pivotal trials of zolmitriptan for migraine. ${ }^{18}$ These included dizziness, paraesthesia, muscle tightness and nausea, occurring in 1-5\% of the study population. ${ }^{8}$ While overall adverse-event reporting rates were much higher in the open-label, long-term safety study (up to 95\%), it should be remembered that participants were queried numerous times during and after attack treatment, over the course of up to 25 attacks, and that skin reactions are to be expected. ${ }^{9}$ The vast majority of adverse events were skin reactions (more than 95\% rated as mild and none rated as severe) that required no treatment, and in addition, systemic reactions were reported at rates below $10 \%$. These included only upper respiratory infection (9.5\%), sinusitis (3.9\%) and nausea (2.4\%), and were not necessarily considered to be treatment related. ${ }^{9}$

\section{Conclusions}

Zolmitriptan has been an attractive option for the acute management of migraine attacks since 1997. Its key attributes lend itself to this role, including rapid absorption via multiple routes, good efficacy and favorable tolerability. Traditionally in headache medicine, most widely accepted therapies are oral formulations, mainly for ease of dosing and familiarity, but there are inherent disadvantages with respect to rapidity of onset of action compared with other routes of administration. Currently, zolmitriptan is available orally and intranasally. The intranasal formulation offers, potentially, more rapid relief, but not all patients find nasal delivery appealing. While other faster routes of delivery, such as by injection or the rectal route, are plausible, they are not currently available, nor are they as widely accepted in American culture. The adhesive, dermally applied, microneedle-array mode of delivery in development has the potential to fill a gap in acute migraine management, by providing a non-oral mode of drug delivery with little to no pain and few moderate or severe adverse events. $]$
1. Olesen J. Headache Classification Committee of the International Headache Society (IHS) The internationa classification of headache disorders, 3rd edition. Cephalalgia. 2018;38:1-211.

2. Stovner $\amalg$, Nichols $E$, Steiner $T$ J, et al. Global, regional, and national burden of migraine and tension-type headache 1990-2016: a systematic analysis for the Global Burden of Disease Study 2016. Lancet Neurol. 2018;17:954-76.

3. Vos T, Abajobir AA, Abbafati C, et al. Global, regional, and national incidence, prevalence, and years lived with disability for 328 diseases and injuries for 195 countries, 1990-2016: A systematic analysis for the Global Burden of Disease Study 2016. Lancet. 2017;390:1211-59.

4. Marmura MJ, Silberstein SD, Schwedt TJ. The acute treatment of migraine in adults: The American Headache Society evidence assessment of migraine pharmacotherapies. Headache. 2015;55:3-20

5. Accessdata.fda.gov. FDA Package Insert ZOMIG (zolmitriptan). 2012. Available at: www.accessdata.fda.gov/drugsatfda_ 2012. Avallable at: WWW.accessdata.fda.gov/drugsattda_docs/label/2012/020768s019s021,0212 label/2012/020768s019s021,021231s010s011/bl.pdf label/2012/020768s019s021,021231s010s011/bl.pdf

6. Accessdata.fda.gov. 2015. Supplement Approval Zom
(zolmitriptan) nasal spray 0.5mg, 2.5mg and 5mg. (zolmitriptan) nasal spray $0.5 \mathrm{mg}, 2.5 \mathrm{mg}$, and $5 \mathrm{mg}$.
Available at: www.accessdata.fda.gov/drugsatfda_docs appletter/2015/0214500rig1s008Itr.pdf (accessed 14 December 2021).

7. Nguyen J, Lewis H, Queja A, et al. Pharmacokinetics and skin tolerability of intracutaneous zolmitriptan delivery in swine using adhesive dermally applied microarray. J Pharm SCi. 2018;107:2192-7.

8. Spierings ELH, Brandes JL, Kudrow DB, et al. Randomized, double-blind, placebo-controlled, parallel-group, multi-center study of the safety and efficacy of ADAM zolmitriptan for the acute treatment of migraine. Cephalalgia. 2018;38:215-24.

9. Nahas SJ, Hindiyeh N, Friedman DI, et al. Long term safety, tolerability, and efficacy of intracutaneous zolmitriptan (M207) in the acute treatment of migraine. J Headache Pain. 2021;22:37.

10. Brunton LL (ed). Goodman \& Gilman's Pharmacological Basis of Therapeutics. 12th edition. New York: McGraw-Hill Education/ Medical. 2011.

11. Chattopadhyay S, Das S, Sarma KN. Nose-to-brain drug delivery: An update to the alternative path to successful targeted antimigraine drugs. Int J Appl Pharm. 2021;13:67-75.

12. Govinfo.gov. 2020. 85 FR 39913 - Teva Branded Pharmaceutical Products R\&D, Inc.; Withdrawal of Approval of a New Drug Application for ZECUITY (Sumatriptan Iontophoretic Transderma Application for ZECUITY (Sumatriptan lontophoretic Transderma
System). Available at: www.govinfo.gov/app/details/FR-2020-07System). Available at: www.govinfo.gov/app/detar

13. Van Der Maaden $K$, Jiskoot W, Bouwstra J. Microneedle technologies for (trans)dermal drug and vaccine delivery. J Control Release 2012;161:645-55.

14. Tepper SJ. Orally inhaled dihydroergotamine: A review. Headache. 2013;53(Suppl.2):43-53.

15. Goadsby PJ, Yates R. Zolmitriptan intranasal: A review of the pharmacokinetics and clinical efficacy. Headache.
2006;46:138-49.

16. Accessdata.fda.gov. 2013. Imitrex Tablets Sumatriptan succinate NDA 020132/S-028 FDA Approved Labeling Text November 2013. Available at: www.accessdata.fda.gov/drugsatfda_docs/ label/2013/020132s028,020626s025lbl.pdf (accessed 14 December 2021).

17. Dixon $R$, Warrander $A$. The clinical pharmacokinetics of zolmitriptan. Cephalalgia. 1997;17(Suppl. 18):15-20

18. Ferrari MD, Goadsby PJ, Roon KI, Lipton RB. Triptans (serotonin 5-HT1B/1D agonists) in migraine: Detailed results and methods of a meta-analysis of 53 trials. Cephalalgia. 2002;22:633-58.

19. Yates R, Nairn K, Dixon R, Seaber E. Preliminary studies of the pharmacokinetics and tolerability of zolmitriptan nasal spray in healthy volunteers. I Clin Pharmacol. 2002;42:1237-43.

20. Accessdata.fda.gov. ZOMIG® (Zolmitriptan) Nasal Spray. 2003. Available at: www.accessdata.fda.gov/drugsatfda_docs/ label/2003/21450_zomig Ibl.pdf (accessed 14 December 2021).

21. Sorensen J, Bergstrom M, Antoni A. Distribution of 11C-zolmitriptan nasal spray assessed by positron emission tomography (PET). Euro J Neurol. 2000;7(Suppl. 3):82.

22. Wall A, Kagedal M, Nilsson D, et al.Distribution of zolmitriptan into the CNS in healthy volunteers: a positron emission tomography study. DrugS R D. 2005;6:139-47.

23. Zingmark P-H, Yates R, Hedlund C, Kagedal M. True nasopharyngeal absorption of zolmitriptan following administration of zolmitriptan nasal spray. Eur I Neurol. 2003;10(Suppl. 1):76. 